\title{
Tracer diffusion and cluster formation in mixtures of spheres and rotating rods
}

\author{
R Kirchhoff and H Löwen \\ Institut für Theoretische Physik II, Heinrich-Heine-Universität Düsseldorf, Universitätsstrasse 1, \\ 40225 Düsseldorf, Germany \\ E-mail: hlowen@thphy.uni-duesseldorf.de
}

Received 8 August 2005

Published 2 December 2005

Online at stacks.iop.org/JPhysCM/17/7805

\begin{abstract}
On the basis of Brownian dynamics computer simulation studies, we investigate the structure and dynamics of two-dimensional mixtures of rods driven by an external torque and spheres. First, we show that the tracer long-time diffusion of spheres in a system of rotating rods can be efficiently tuned via the rod density. It behaves non-monotonically for increasing rod density. In particular, the sphere diffusion becomes strongly enhanced across the jamming transition of the rods. Second, we show that rotating rods in a dense suspension of spheres form aggregates of rod clusters which are rotating jointly. The cluster size is in reasonable agreement with the predictions of a simple theory.
\end{abstract}

(Some figures in this article are in colour only in the electronic version)

\section{Introduction}

When exposed to circularly polarized light, anisotropic colloidal particles experience a driving torque which induces a particle rotation [1-4]. The rotation is damped in the viscous solvent. The magnitude of the driving torque can be tuned via the intensity of the light field. This introduces novel effects and opens up interesting possibilities of externally controllable mixers in microfluidic devices [5-7]. These rotating colloids furthermore constitute simple examples for self-propelled [8] or active Brownian particles [9-11] as well as Brownian motors [12].

In this paper we explore, by means of Brownian dynamics computer simulations, the dynamics of spherical tracer particles [13] in a concentrated suspension of interacting driven rods [14]. An ensemble of dense driven rotators was recently shown [15] to perform a jamming transition from a T-structure where neighbouring rods are oriented perpendicular to each other to a jammed configuration with rods preferentially oriented parallel to each other. This nonequilibrium phase transition in the steady state is expected to have a drastic influence on the dynamics of small tracer particles embedded in the suspension of rotating rods. In this paper we show that-perhaps counterintuitively - the tracer dynamics becomes faster when the rotators 
get jammed. This dramatic increase is due to the fact that tracers are kept in voids at lower densities when the rods favour a T-structure. These results show that tracer dynamics can be tailored by external control which might be useful for several applications in fabricating microfluidic devices.

We further investigate self-organization of driven rods in a concentrated suspension of spheres. This case is opposite to that just discussed in that now the concentration of spheres is large. Starting from a configuration where spheres and rods are completely mixed, the rotating rods tend to form clusters (or aggregates) performing a joint rotation. This reduces the osmotic pressure needed to create a void in the dense sphere suspension. The number of the rods participating in the same aggregate (the so-called aggregate size), however, is finite, since the rods repel each other. Our computer simulation data for the mean aggregate size are in line with a simple thermodynamic theory.

Our data are obtained within a simple two-dimensional model of rod-sphere mixtures. The rods are composed of spherical beads and all beads and spheres interact via a repulsive Yukawa pair potential which describes the effective interaction between charged suspensions [16]. The rods and spheres move according to simple totally overdamped Brownian dynamics and hydrodynamic interactions mediated by the solvent are neglected. Colloidal mixtures of rods and spheres have been studied before both in equilibrium [17-21] and in non-equilibrium [22]. Our two-dimensional model can be realized by confining the particles to two dimensions either using an optical tweezer or in a pendent liquid-gas interface [23].

The paper is organized as follows. In section 2 we describe the interactions and the dynamics of our model and the computer simulation techniques. Results for the long-time diffusion of tracer spheres in concentrated suspensions of driven rods are presented in section 3 . The converse case, namely driven rods in a dense suspensions of spheres, is considered in section 4 where data for the cluster formation are described. We finally conclude in section 5 .

\section{The model}

Our model comprises a two-dimensional binary mixture of driven rods and non-driven spheres. We consider $N_{1}$ two-dimensional rod-like particles moving in an area $A$ which are characterized by their centre of mass and their orientation. In addition to the rods, we include $N_{2}$ spherical particles characterized by their centre of mass.

The rods interact via a Yukawa segment model which was shown to be a good representation of charged rod-like colloids [24]. There are $N_{\mathrm{b}}=10$ equidistant beads along the rods; the bead spacing along the rods is given by the bead diameter $\sigma$, which sets the total physical rod length as $L=N_{\mathrm{b}} \sigma$. The bead diameter $\sigma$ sets the physical diameter of the rods and serves as a natural length scale. The repulsive interaction potential between beads of different rods is

$$
V(r)=U_{0} \sigma \frac{\exp [-\kappa(r-\sigma) / \sigma]}{r}
$$

where $r$ is the inter-bead distance, $U_{0}$ is an energy scale and $\kappa$ is the dimensionless screening parameter. The number density of the rods is specified in terms of the dimensionless area packing fraction $\eta=\pi N_{1} L^{2} / 4 A$. The rods are kept at a constant temperature $T$.

The rods move according to Brownian dynamics [25] with rotational friction $\gamma_{\mathrm{R}}$ and anisotropic translational frictions $\gamma_{\|}$and $\gamma_{\perp}$, parallel and perpendicular to their orientations [25]. These friction coefficients were taken from [26] for a rod aspect ratio of 10 and a rod diameter $\sigma$. The standard Langevin dynamics with neglected hydrodynamic interactions was adopted. Random kicks of the solvent keep the suspension of rods at constant thermal energy $k_{\mathrm{B}} T$. On top of that the rods are exposed to a constant external torque $D$ which 
tends to rotate all rods in the same direction, driving the system into a non-equilibrium steady state [15]. The effect of the torque on a single rod at zero temperature $(T=0)$ is that it leads to a constant angular velocity

$$
\omega=\gamma_{R} D \text {. }
$$

Together with the rods, there are $N_{2}$ spheres in the system corresponding to a number density $\rho_{2}=N_{2} / A$. For simplicity, unless otherwise stated, the sphere-sphere and rod-sphere interaction is taken to be identical to that between beads-see equation (1)-i.e. a sphere is identical to an isolated bead and has a physical diameter $\sigma$. The translational dynamics of the spheres is simple Brownian dynamics again with the corresponding Stokes friction coefficient $\gamma$. The diffusion coefficient of a single free sphere is therefore

$$
D_{0}=k_{\mathrm{B}} T / \gamma \text {. }
$$

The spheres do not feel any external force or torque.

We have solved the Langevin equation for the spheres and the rod centres and orientations in a Brownian dynamics computer simulation with a finite time step $\Delta t=0.009 \tau_{\mathrm{B}}$, where $\tau_{\mathrm{B}}=\gamma_{\|} \sigma^{2} / U_{0}$ is a suitable Brownian timescale. The rods are in a quadratic box with periodic boundary conditions in $x$ and $y$ directions. Typically we simulated $N=80-200$ rods and 1-100 spheres for 300000 time steps to get into the steady state. Finite size effects were carefully checked. Statistics in the steady state were gathered by a further time averaging over typically 100000 time steps. In our simulations we fixed the external torque to $D / U_{0}=150$, the reduced temperature $T^{*}=k_{\mathrm{B}} T / U_{0}$ to 0.1 and the screening parameter to $\kappa=2$. We varied the rod and sphere densities.

\section{Long-time diffusion of tracer spheres in rotating rods}

First let us consider the case of a single tracer sphere in a concentrated suspension of rotating rods. The long-time dynamics of the single tracer particles is characterized by the long-time translational self-diffusion coefficient $D_{\mathrm{s}}$ :

$$
D_{\mathrm{s}}=\lim _{t \rightarrow \infty} \frac{1}{4 t}\left\langle\left(\vec{r}^{(\mathrm{s})}(t)-\vec{r}^{(\mathrm{s})}(0)\right)^{2}\right\rangle
$$

where $\vec{r}^{(\mathrm{s})}(t)$ denotes the time-dependent trajectory of the sphere and $\langle\cdots\rangle$ is a time average. The tracer diffusion coefficient is a function of the temperature and the rod density. Obviously, at high rod dilution $\left(\eta \rightarrow 0\right.$ ) or at high temperature, $D_{\mathrm{s}}$ equals the free sphere diffusion constant $D_{0}$. Consequently, the ratio $D_{\mathrm{s}} / D_{0}$ gives information about whether the dynamics of the tracer particle is accelerated or hindered by the presence of the rotating rods.

Before discussing the results of our computer simulations, let us do a simple length and timescale analysis. The averaged rotation time for a single rod is $2 \pi / \omega$ and during this time a single free sphere has diffused over a distance of about

$$
b=\sqrt{D_{0} 2 \pi / \omega}
$$

The ratio $\alpha=b / L$ tells us how far the single sphere can penetrate into neighbouring rotating rods during one rotation period. For the parameters investigated in our work, $\alpha=0.025 \ll 1$; hence the diffusing spheres will only feel the outermost beads of the rotating beads which hinders the penetration of the sphere into the area covered by the rotating rod.

Simulation data for $D_{\mathrm{s}} / D_{0}$ as a function of the rod packing fraction $\eta$ are presented in figure 1. The inset shows that the tracer long-time diffusion decreases with increasing rod density. In principal, there are two competing effects: the presence of the rotating rods provides geometrical constraints for the sphere diffusion; hence the dynamics is slowed down. 


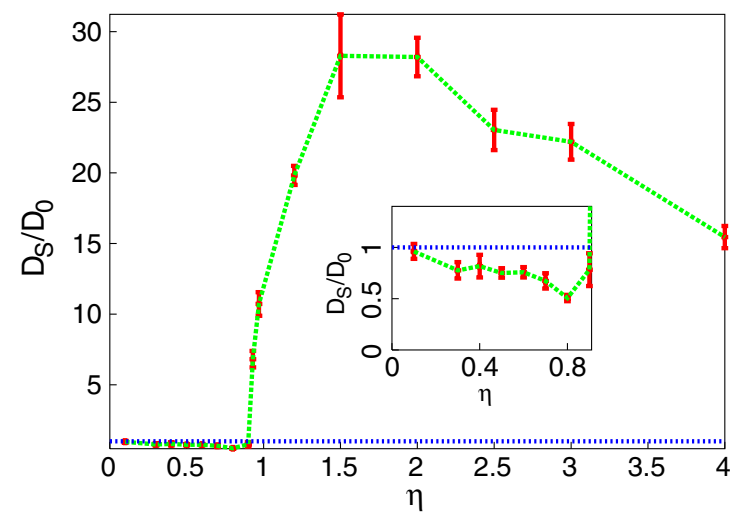

Figure 1. The long-time sphere self-diffusion coefficient $D_{\mathrm{s}}$, measured in terms of its free diffusion constant $D_{0}$, as a function of the dimensionless area rod density $\eta$. The dotted line denotes the case $D_{\mathrm{s}}=D_{0}$ as a reference. The inset is an enlargement of the same data for small $\eta$. The jamming transition of the pure Brownian rotator occurs at $\eta=0.95$ [15].

On the other hand, the rotative motion of the rods may push a sphere, thus enhancing $D_{\mathrm{s}}$. In our case the rotating rods constitute dynamical repulsive obstacles for the sphere motion due to the smallness of the $\alpha$ parameter. Therefore the diffusion of the sphere is slowed down to about $50 \%$ relative to the free sphere case for low rod area fractions $\eta .^{1}$

Then at about $\eta \approx 0.9$ there is a drastic increase in $D_{\mathrm{s}}$. This coincides with the location of the rod jamming transition which occurs at $\eta=0.95$ [15]. Near jamming the orientation correlation of neighbouring rotating rods changes dramatically from perpendicular orientation (so-called T-structure) to parallel orientation. While the T-structure efficiently confines the spheres for small $\alpha$ leading to a cage effect, the collective motion of parallel rods provokes an escape from the spheres from these cages. Interestingly the dynamics of the sphere gets faster although the rod dynamics is becoming jammed due to mutual hindrance of their rotation. At high rod densities again, the rod jamming is becoming so severe that it also blocks the sphere diffusion; hence $D_{\mathrm{s}}$ decreases with increasing $\eta$. The relevant mechanisms are sketched in figure 2 .

This general scenario is supported by typical simulation snapshots in the steady state of the system as shown in figure 3. For $\eta=0.8$, i.e. for a clear T-structured rod configuration below jamming, tracer spheres sit preferentially in the centres of the jointly rotating neighbouring rods as indicated by the circles in figure 3(a). Above the jamming transition at $\eta=1.2$, the sphere is pushed and accelerated by the rotating rod cluster; see figure 3(b). Increasing $\eta$ further will result in blocking of the sphere's motion by jammed clusters; see figure 3(c).

In conclusion we have shown that the dynamics of tracer particles is drastically affected by the kinetics of the embedding phase. For a rotating rod suspension, the dynamics can be enhanced by several orders of magnitude if the jamming transition of the rods is reached. This gives immediate external control to the dynamics of tracer particle via external fields.

\section{Rotating rod aggregates in concentrated sphere suspensions}

Let us now discuss the opposite case of high sphere density; then the rods are dispersed in a dense suspension of spheres. We consider several cases for the rod-rod interaction, the first

1 The opposite trend is expected for $\alpha$ parameters which are of the order of or larger than unity since here the sphere can penetrate into the region covered by the rotating rods and is thus efficiently propelled further. 


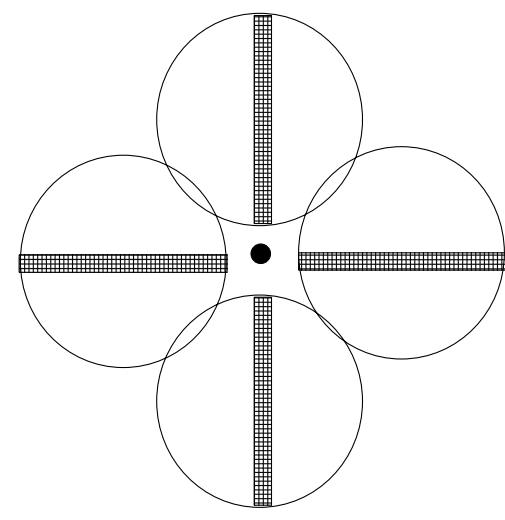

(a)

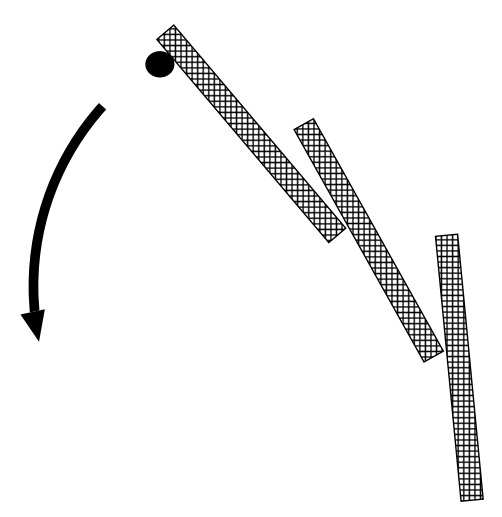

(b)

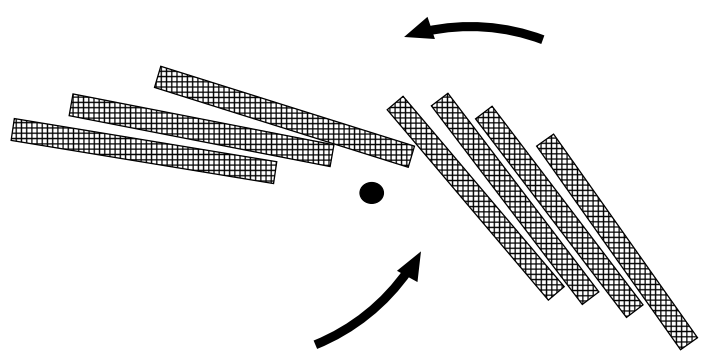

(c)

Figure 2. Schematic view of the different mechanisms relevant for the diffusion of a tracer sphere in a rotating rod suspension for three different domains of the rod areal packing fraction $\eta$. (a) For $\eta \approx 0.8$, the sphere feels the rotating rods as effective repulsive circles with radius $L$. This leads to a caging effect in a quadruple of rotating rods with a relative T-structure. The circles around the rods indicate the area covered during one period of rod rotation. (b) Close to rod jamming $(0.9 \leqslant \eta \lesssim 1.5)$, the sphere collides frequently with a rotating cluster of rods which pushes the sphere forwards and thus accelerates the sphere dynamics. The arrow indicates the overall motion of the rod cluster. (c) For large $\eta, \eta \gtrsim 1.5$, the rod jamming is severe and again slows down the dynamics of the sphere. The arrows indicate the overall motion of the rod cluster.

of which is identical to the previously assumed Yukawa segment interaction. We find that the rods will aggregate into rotating clusters since their rotation excludes free accessible area for the spheres and this volume is minimized if rods are clustering together. During the simulation, the number of rods participating in a cluster changes, ensuring that the clusters form the steady state of the system. The intra-cluster structure depends on details of the rod-rod interaction.

\subsection{Repulsive Yukawa segment rod-rod interaction}

In a dense suspension of spheres, rotating rods will tend to aggregate. We consider typical sphere densities $\rho_{2} \sigma^{2}$ of the order of $0.26-0.74$. The rod centre-centre pair distribution function $g(r) \equiv g_{11}(r)$ is shown for three different sphere densities $\rho_{2} \sigma^{2}$ in figure 4 . In addition to the 'normal' peak at a mean inter-rod spacing of about $r \approx L$, there is a pre-peak at a distance of $r=\sigma$ which signals the tendency of neighbouring rods to align and form a joint cluster. The sharpness of this cluster peak increases with increasing $\rho_{2}$. 

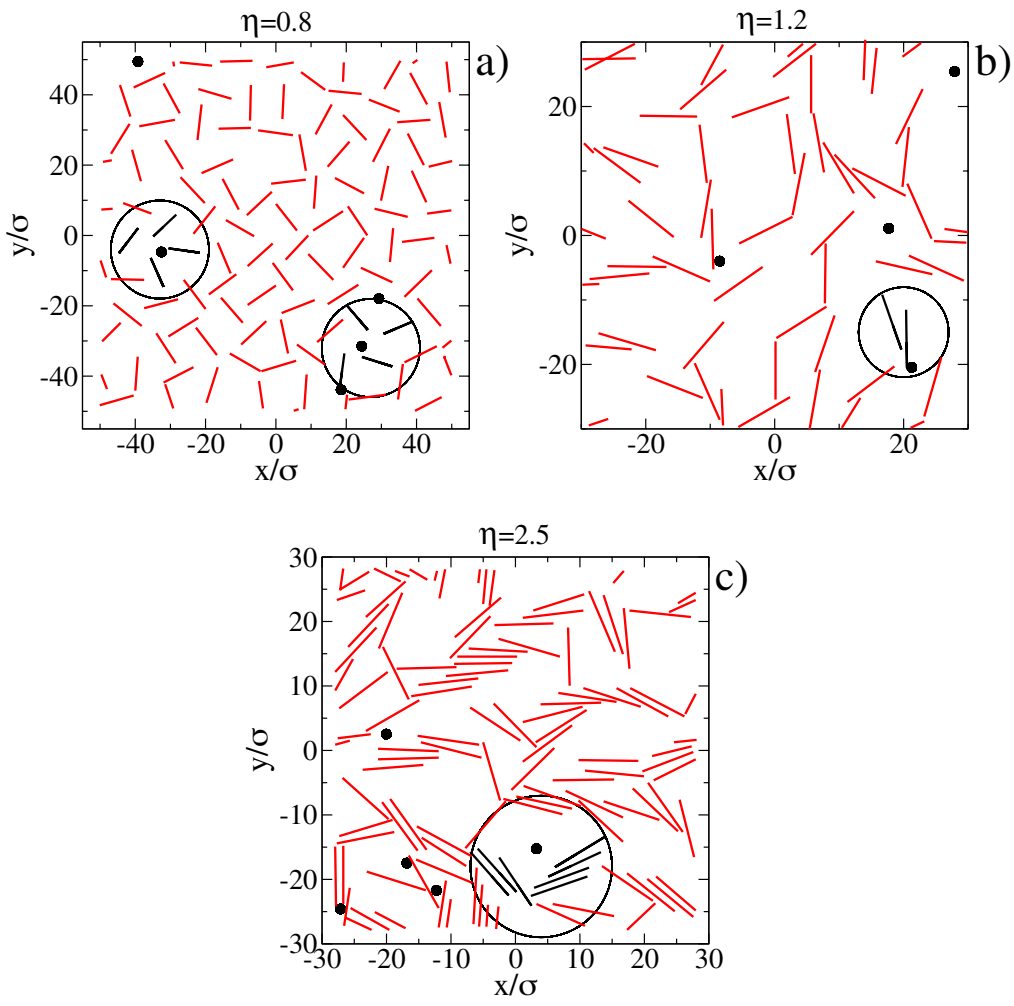

Figure 3. Typical simulation snapshots in the steady state of a binary mixture of driven rods and spheres. The density of the spheres is finite-namely five particles per simulation box area-but the sphere density is so dilute that the spheres are effectively non-interacting with each other. The position of the spheres is indicated by black circles. (a) For $\eta=0.8$, there is a T-structure visible in the driven rod system. This density is slightly below the jamming transition. The spheres are preferentially locked in the area not covered by neighbouring rotating rods as indicated schematically in figure 2(a). The corresponding rods participating in the joint caging of the spheres are included in the two circles shown. (b) $\eta=1.2$, slightly above the jamming transition of the rods. The circle denotes a situation where the sphere is pushed by the anticlockwise rotating rod cluster. (c) $\eta=2.5$, well above the jamming transition of the rods. The circle visualizes a situation where the sphere motion is blocked by anticlockwise rotating rod clusters. Increasing the rod density further will slow down the sphere dynamics even more.

A simulation snapshot in the steady state for a high sphere density of $\rho_{2} \sigma^{2}=0.37$ is shown in figure 5 which clearly indicates that several pairs of aligned rods are forming clusters which are propelled jointly by the external torque. Still there are some single rods rotating alone. The area inaccessible for the spheres created by the anticlockwise rod rotation is clearly seen.

We have further investigated a model where half of the rods are rotating anticlockwise and the other half are rotating clockwise; see figure 6 for a simulation snapshot. Clearly only two rods which are rotating in the same direction are participating in the same cluster.

\subsection{Non-interacting rods}

The next extreme case is that of vanishing rod-rod interaction. This is interesting as a limiting reference system. Here all the rods simulated self-organize into a single cluster. 

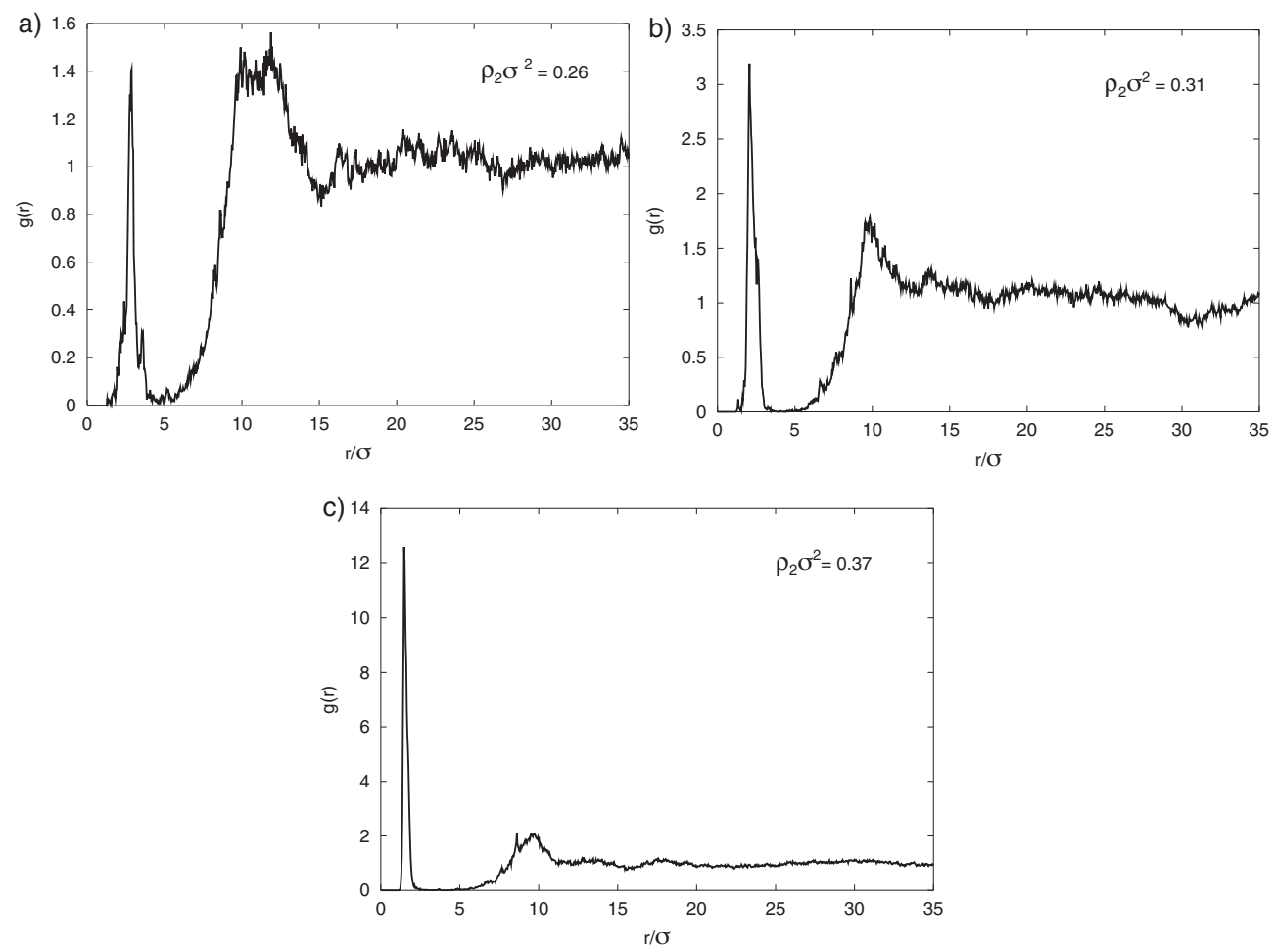

Figure 4. Rod centre-centre pair distribution function $g(r)$ for three different sphere densities: (a) $\rho_{2} \sigma^{2}=0.26$, (b) $\rho_{2} \sigma^{2}=0.31$, (c) $\rho_{2} \sigma^{2}=0.37$. The sharp cluster pre-peak at $r \approx 2 \sigma$ is clearly visible. The rod density is $\eta=0.8$.

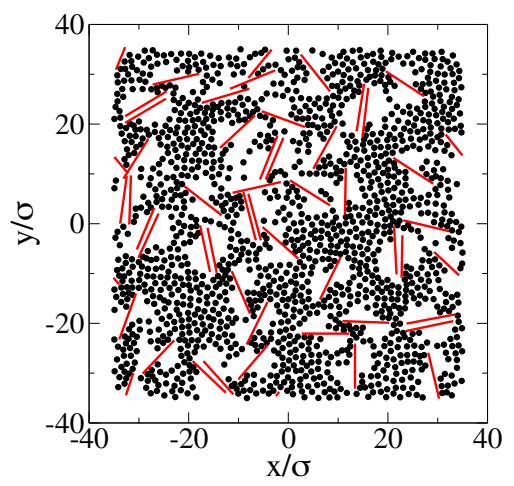

Figure 5. Typical simulation snapshot for a rotating rod system in a suspension of dense spheres. The rotation of the rods is anticlockwise. The position of the spheres is denoted with black circles. The Yukawa segments of the rods are shown as crosses. The sphere density is $\rho_{2} \sigma^{2}=0.37$ while the rod density is $\eta=0.8$.

This is obviously the configuration that minimizes the area inaccessible to the spheres. The configuration after a long simulation run is depicted in figure 7. 


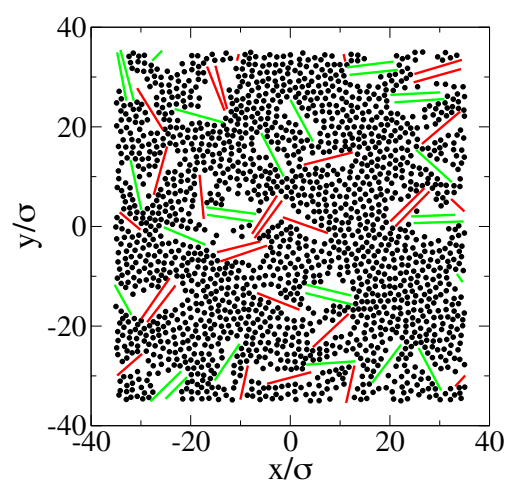

Figure 6. As figure 5, but now one half of the rods are rotating clockwise (light grey) an the other half anticlockwise (dark grey).

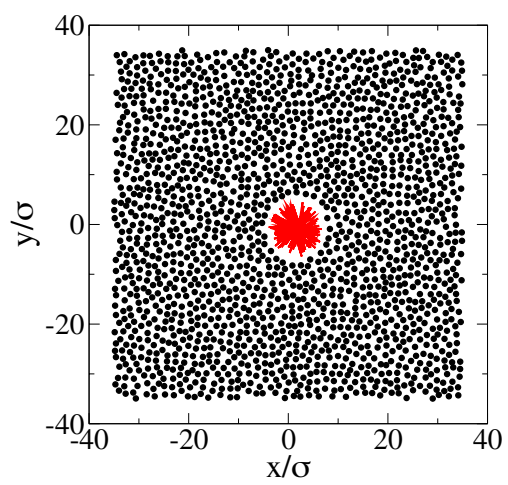

Figure 7. Typical simulation snapshot for a non-interacting rod system in a suspension of dense spheres. A single rod cluster is formed. The total simulation time was $6000 \tau_{\mathrm{B}}$. The sphere density is $\rho_{2} \sigma^{2}=0.74$ while the rod density is $\eta=0.8$.

\subsection{Rod dumb-bell model}

In the so-called rod dumb-bell model only the two outermost segments of the rods are interacting with each other. The inner segments just contribute to the rod-sphere interaction. Here the system self-organizes into several clusters and the inner cluster structure is star-like, which results from the dumb-bell-like repulsion of the rods. A typical snapshot gained after a long simulation is presented in figure 8. The aggregate size (i.e. the number of rods contributing to a cluster) is not sharply distributed, but there is a clear averaged (mean) value $N_{\mathrm{c}}$.

\subsection{Theory for the mean aggregate size}

We finally present a variational theory for the mean aggregate size $N_{\mathrm{c}}$ based on simple energy considerations of a cluster. Basically there is a competition between two effects. The osmotic bulk pressure $p_{2}$ of the spheres tends to push all rods into a single cluster as was shown in the reference model studied in section 4.1. If the angular velocity $\omega$ is fast relative to the sphere motion (or if, in other words, the $\alpha$ parameter introduced in section 3 is small), a single rotating rod generates an area of $\pi L^{2} / 4$ which cannot be occupied by the spheres. Hence this corresponds roughly to a free energy cost of $p_{2} \pi L^{2} / 4$. If aggregates are present which contain 


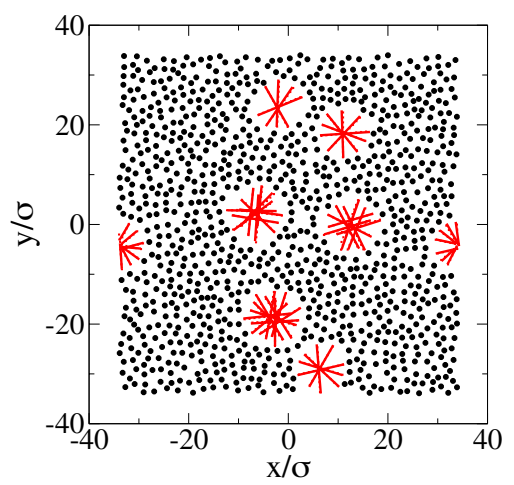

Figure 8. Typical simulation snapshot for the rod dumb-bell model. Seven rod clusters are formed. The total simulation time was $6000 \tau_{\mathrm{B}}$. The sphere density is $\rho_{2} \sigma^{2}=0.37$ while the rod density is $\eta=0.8$.

exactly $N_{\mathrm{c}}$ rods, this free energy penalty has to be multiplied by the number of aggregates $N_{1} / N_{\mathrm{c}}$ with $N_{1}$ denoting the total number of rods. This energy contribution favours large cluster sizes.

The second contribution summarizes the potential energy penalty $u\left(N_{\mathrm{c}}\right)$ within a single cluster. Clearly, $u\left(N_{\mathrm{c}}\right)$ is zero for the non-interacting rods. For the dumb-bell model we assume star-like aggregates; therefore

$$
f\left(N_{\mathrm{c}}\right)=\sum_{i<j} V\left(\left|\vec{r}_{i}-\vec{r}_{j}\right|\right)
$$

with

$$
\vec{r}_{i}= \pm L\left(\begin{array}{c}
\cos \phi_{i} \\
\sin \phi_{i}
\end{array}\right) .
$$

The total free energy of the system is

$$
F\left(N_{\mathrm{c}}\right)=\frac{N_{1}}{N_{\mathrm{c}}} p_{2} \pi L^{2} / 4+\frac{N_{1}}{N_{\mathrm{c}}} u\left(N_{\mathrm{c}}\right) .
$$

Minimizing $F\left(N_{\mathrm{c}}\right)$ with respect to $N_{\mathrm{c}}$ yields the realized mean cluster size. The bulk sphere pressure $p_{2}$ was obtained by a reference calculation in a rod-free system via the virial rule [27]; the corresponding data are shown as a function of sphere density $\rho_{2}$ in figure 9. Clearly for the non-interacting rod case, the theory predicts $N_{\mathrm{c}}=\infty$ in agreement with our findings of section 4.2 .

For the dumb-bell model, theoretical results as obtained by minimization of the expression (8) for the cluster size $N_{\mathrm{c}}$ realized are shown in figure 10 versus the sphere density $\rho_{2}$. They are compared with extensive simulation studies. The simulation snapshots do not yield a monodisperse distribution of $N_{\mathrm{c}}$ but it is their mean number which is compared to the theory. Another caveat is that at finite rod concentration, the number density of the spheres which has to be inserted in $p_{2}\left(\rho_{2}\right)$ has to be rescaled towards a higher effective density $\rho_{2}^{*}=N_{2} /\left(A-\frac{N_{1}}{N_{c}} \pi L^{2} / 4\right)$. Figure 10 shows that the comparison performs quite well for small sphere densities but for high densities there are systematic deviations which we attribute to the fact that the simulations have possibly not yet converged to the final steady state. The deviation may also be due to the severe approximations involved in our variational theory. 


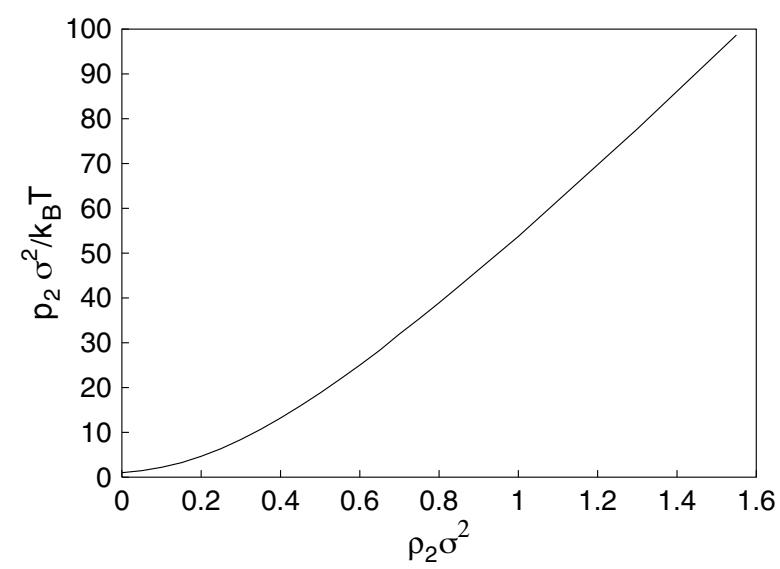

Figure 9. Reduced bulk osmotic pressure (or equation of state) $p_{2}\left(\rho_{2}\right) \sigma^{2} / k_{\mathrm{B}} T$ for a twodimensional Yukawa system versus reduced density $\rho_{2} \sigma^{2}$.

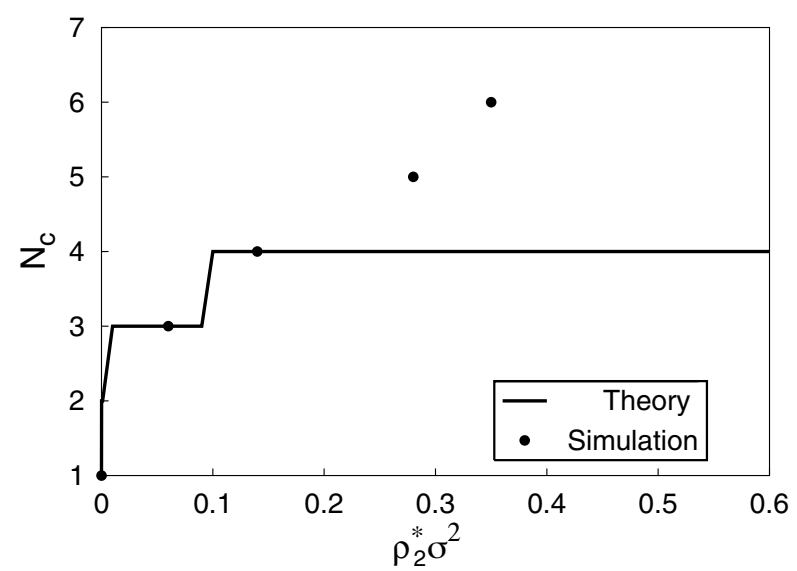

Figure 10. Mean aggregate size $N_{\mathrm{c}}$ versus effective sphere density $\rho_{2}^{*}$. The solid line is the prediction based on the variational theory. The dots are computer simulation results.

\section{Conclusions}

In conclusion, we have studied tracer diffusion and cluster formation in mixtures of driven rods and undriven spheres and found a variety of non-equilibrium phenomena. Across the jamming transition of the rods, the dynamics of the spherical tracer particle additives is enhanced. This may be exploited to tune the mixing effects in microfluidic devices [5, 6]. Furthermore, in dense suspension, rotating rods aggregate together into collectively rotating clusters. If the rod interaction is concentrated on an repulsive dumb-bell, rotating stars are found as stable steady states. The number of rods participating in the aggregate can be tailored by e.g. the osmotic pressure of the suspending sphere dispersion.

Future work should first focus on an experimental verification of our theoretical predictions. The system can be realized in confined colloidal suspensions of charged [23] or magnetic particles [28], as well as in dusty plasmas [29].

Furthermore, hydrodynamic effects mediated by solvent flow [30] are neglected in our approach which is based on simple Brownian dynamics with Stokes flow. The influence of 
hydrodynamic interactions should be considered thoroughly in more detail in future work, although for very thin rods they seem to be negligible [31].

Finally it would be interesting to expose the spheres also to a driving force. In the rodfree case, lane formation and interfacial instabilities were observed in such driven binary mixtures [32-35]. One would expect a huge variety of new non-equilibrium effects in combining a translational drive on the spheres with an orientational drive on the rods.

\section{Acknowledgments}

We thank A Wysocki, H H Wensink and P Johnson for interesting discussions. We thank L Assoud for helpful remarks. This work is supported by the Deutsche Forschungsgemeinschaft (SFB TR6), by the EU Marie-Curie network 'Dynamical Arrest' (proposal No 504712), and by the EU network SOFTCOMP (proposal No 502235-2).

\section{References}

[1] Cheng Z, Chaikin P M and Mason T G 2002 Phys. Rev. Lett. 89108303

[2] Sullivan M, Zhao K, Harrison C, Austin R H, Megens M, Hollingsworth A, Russel W B, Cheng Z, Mason T and Chaikin P M 2003 J. Phys.: Condens. Matter 15 S11

[3] Ashkin A 1992 Biophys. J. 61 569-82

[4] Bishop A I, Nieminen T A, Heckenberg N R and Rubinsztein-Dunlop H 2003 Phys. Rev. A 68033802

[5] Bertsch A, Heimgartner S, Cousseau P and Renaud P 2001 Lab on a Chip 156

[6] Lu L H, Ryu K S and Liu C 2002 J. Microelectromech. Syst. 11462

[7] Bishop A I, Nieminen T A, Heckenberg N R and Rubinsztein-Dunlop H 2004 Phys. Rev. Lett. 92198104

[8] Ebeling W 2004 Solid State Phenom. 97/98 37

[9] Toner J and Tu Y 1998 Phys. Rev. E 584828

[10] Hatwalne Y, Ramaswamy S, Rao M and Simha R A 2004 Phys. Rev. Lett. 92118101

[11] For a recent review, see Schweizer F 2003 Brownian Agents and Active Particles: Collective Dynamics in the Natural and Social Sciences (Springer Series in Synergetics vol XVI) (Berlin: Springer) (ISBN: 3-54043938-2)

[12] Reimann P and Hänggi P 2002 Appl. Phys. A 75169

[13] Nägele G and Dhont J K G 1998 J. Chem. Phys. 1089566

[14] Löwen H 2001 J. Phys.: Condens. Matter 13 R415

[15] Kirchhoff R and Löwen H 2005 Europhys. Lett. 69291

[16] Hansen J P and Löwen H 2000 Annu. Rev. Phys. Chem. 51209

[17] Adams M, Dogic Z, Keller S L and Fraden S 1998 Nature 393349

[18] Chen Y L and Schweizer K S 2002 J. Chem. Phys. 1171351

[19] Schmidt M and Denton A R 2002 Phys. Rev. E 65021508

[20] Zhang S D, Reynolds P A and Van Duijneveldt J S 2002 Mol. Phys. 1003041

[21] Brader J M, Evans R and Schmidt M 2003 Mol. Phys. 1013349

[22] Dhont J K G and Briels W J 2003 J. Chem. Phys. 1181466

[23] Johnson P and van Blaaderen A, private communication

[24] Löwen H 1994 Phys. Rev. Lett. 72424 Löwen H 1994 J. Chem. Phys. 1006738

[25] Three-dimensional Brownian dynamics simulation in equilibrium are studied in Kirchhoff T, Löwen H and Klein R 1996 Phys. Rev. E 535011

[26] Tirado M M, Martinez C L and de la Torre J G 1984 J. Chem. Phys. 812047

[27] Löwen H 1992 J. Phys.: Condens. Matter 410105

[28] Zahn K and Maret G 1999 Curr. Opin. Colloid Interface Sci. 460

[29] Salimullah M, Shukla P K, Sandberg I and Morfill G E 2003 New J. Phys. 540

[30] Nägele G 1996 Phys. Rep. 272216

[31] Dhont J K G and Briels W J 2003 J. Chem. Phys. 1181466

[32] Dzubiella J, Hoffmann G P and Löwen H 2002 Phys. Rev. E 65021402

[33] Chakrabarti J, Dzubiella J and Löwen H 2003 Europhys. Lett. 61415

[34] Chakrabarti J, Dzubiella J and Löwen H 2004 Phys. Rev. E 70012401

[35] Wysocki A and Löwen H 2004 J. Phys.: Condens. Matter 167209 